\title{
Gasometría y tolerancia clínica de los pacientes durante hemodiálisis con baños de bicarbonato y de acetato de sodio.
}

Gasometry and clinical tolerance of patients during hemodyalisis with sodium bicarbonate and acetate.

\author{
CIEZA Javier', HUAMAN Carlos ${ }^{1}$, ESTREMADOYRO Luis ${ }^{1}$ \\ ${ }^{1}$ Servicio de Nefrología de la Universidad Peruana Cayetano Heredia.
}

\section{SUMMARY}

Hemodialysis is more phsiological when bicarbonate is used in the bath instead of acetate. The new hemodialysis techniques (high efficiency and high flux) need also a bicarbonate dialysis bath. The influence of the gasometric changes on th hemodialysis related sintomatology has not been adecuately described. In this paper we compare the gasometric evolution in 56 hemodialysis of three hours duration, done in 14 patients (7 high symptomatic and 7 oligosymptomatic), Each one received two sessions with bicarbonate dialysis bath and two sessions with acetate bath. Hemodialysis related symptomatology was absent when bicarbonate dialysis bath was used. Blood bicarbonate and pH got normal levels earlier, and hipoxemia was less severe with bicarbonate bath compared to acetate bath. (Rev Med Hered 1993; 4(4): 173-177)

KEY WORDS: Hemodialysis, bicarbonate dialysate, blood gas

\section{RESUMEN}

La hemodiálisis es más fisiológica cuando se usa baño de bicarbonato en vez de acetato y las nuevas técnicas de hemodiálisis (alta eficiencia y alto flujo), requieren del uso de estos baños. La influencia de los cambios en la gasometría sobre la sintomatología en hemodiálisis de pacientes con características especiales, no ha sido adecuadamente descrita. En el presente trabajo, comparamos la evolución de la gasometría arterial en un grupo de 6 hemodiálisis realizadas en 14 pacientes ( 7 altamente sintomáticos y 7 oligo-sintomáticos), tratados en dos sesiones con baño de bicarbonato y dos con baño de acetato de 3 horas cada una. (Rev Med Hered 1993; 4(4): 165-172)

PALABRAS CLAVE: Tétanos, factores de pronóstico, clasificación de pronóstico. 


\section{INTRODUCCIÓN}

Los programas de hemodiálisis crónica intermitente (HDCI) permiten actualmente largos períodos de sobrevida para los pacientes con insuficiencia renal crónica terminal (IRCT) $(1,2,3,4)$. Esta mejora de la sobrevida ha implicado la aparición de nueva patología como la amiloidosis en diálisis $(5,6)$, y la necesidad de hacer al procedimiento más fisiológico para mejorar la tolerancia hemodinámica y la sintomatología en hemodiálisis $(7,8,9,10)$. Es también importante lograr un adecuado control sobre la acidosis metabólica para ejercer prevención secundaria sobre la osteodistrofia renal (6).

Cuando se iniciaron las experiencias con hemodiálisis, se usaron soluciones dializantes que tenían bicarbonato como sustancia base $(6,8)$. Estas soluciones presentaban el inconveniente de la precipitación de sales de calcio y magnesio, por lo que cuando se iniciaron los programas de HDCI para grandes poblaciones durante las décadas de los $60^{\prime} \mathrm{y}$ 70 's $(6,7)$, fueron reemplazadas por baños de acetato. El reconocimiento de una mejor tolerancia hemodinámica y sintomatológicamente asociada a un mejor control de la acidosis metabólica, menor riesgo de hipoxemia, de vasodilatación y cardiodepresión, han hecho que en la actualidad se recomiende el uso rutinario de baños de bicarbonato $(6,11)$. El acortamiento del tiempo de diálisis por las técnicas de alta eficiencia y alto flujo, que aumentan significativamente la tranferencia de acetato, requieren necesariamente de baños con bicarbonato $(6,7)$.

En nuestro país una gran magnitud de los servicios de hemodiálisis se realizan en sistemas de tanque central donde la preparación de baños de bicarbonato puede resultar en un problema técnico. De otro lado, el equipamiento de los centros con máquinas proporcionadotas de bicarbonato resulta de difícil solución económica para países con dificultades económicas como el nuestro. Nosotros nos proponemos describir en este trabajo una técnica sencilla y de bajo costo para la preparación de soluciones dializantes que contengan bicarbonato como base.

Es objetivo del presente trabajo comparar la evolución de los gases arteriales de pacientes dializados con baños de acetato y de bicarbonato, así como la tolerancia sintomática y hemodinámica.

\section{PACIENTES Y MÉTODOS}

El presente trabajo se realizó en el centro de diálisis particular Servicios Médicos Corpac S.A, Lima-Perú en el mes de Abril de 1993. Se seleccionaron 7 pacientes sintomáticos en HDCI cuyo criterio fue presentar hipotensión arterial sintomática de alta frecuencia durante sus últimas 12 sesiones ( $>7$ hipotensiones tratadas) y/o sintomatología de alta frecuencia ( $>7$ sesiones sintomáticas en sus últimas 12 diálisis). Se consideraron síntomas asociados a HDCI, cefalea y náuseas y vómitos. Se definió como hipotensión arterial sintomática a la caída de la presión arterial sistólica basal $\geq 20 \mathrm{mmHg}$ y que se acompañó por cualquiera de los síntomas asociados. La valoración de los síntomas se hizo como presencia o ausencia. 
Otros 7 pacientes fueron seleccionados entre aquellos que no presentaron hipotensión arterial ni sintomatología en sus últimas 12 sesiones de hemodiálisis.

En todas las sesiones se usaron filtros convencionales de hemodiálisis de membrana de cuprophan de $1.5 \mathrm{~m}^{2}$ de área.

Todos los pacientes fueron observados en 4 sesiones durante dos semanas consecutivas. En cada semana se evaluaron la segunda y tercera sesión. Por baloteo cada paciente se dializó secuencialmente una semana en baño de bicarbonato o acetato y la siguiente semana en baño de acetato o bicarbonato respectivamente. De este modo cada paciente recibió dos diálisis con acetato y dos con bicarbonato y sirvió como su propio control. En total se evaluaron 56 sesiones de hemodiálisis.

En cada sesión se midió $\mathrm{pH}$, bicarbonato, $\mathrm{pCO}_{2}, \mathrm{CO}_{2}$ total $\mathrm{pO}_{2}$ arterial en muestras tomadas inmediatamente antes de iniciar la sesión, a la primera, segunda y tercera hora de hemodiálisis y una hora después de terminado el procedimiento. Se usó un analizador de gases arteriales Ciba-Cornig. Igualmente se registraron las ocurrencias de sintomatología, curso de las funciones vitales cada 15 minutos y volumen de solución salina administrada a cada paciente para mantener la presión arterial estable cuando ésta disminuyó.

Se preparó una solución concentrada, que al ser diluida posteriormente en 34 partes de agua deionizada y acidificada hasta un $\mathrm{pH}$ entre 3.5-3.7, aportó las siguientes concentraciones de sales: Sodio: 140mEq/L; Cloro: $106 \mathrm{mEq} / \mathrm{L} ;$ Potasio: $1.9 \mathrm{mEq} / \mathrm{L}$; Calcio:7.4mg/dl; Magnesio:1.5mEq/L. A esta solución se agregó bicarbonato de sodio USP (300GR) para obtener una concentración de bicarbonato de $34 \mathrm{mEq} / \mathrm{L}$.

Esta solución final pasó por un filtro de 2 micrones de tamano de poro, para servir de inmediato como solución dializante.

Las concentraciones electrolíticas de las soluciones de acetato fueron: Sodio: $140 \mathrm{mEq} / \mathrm{L}$; Cloro: $110 \mathrm{mEq} / \mathrm{L}$; Potasio: $1.8 \mathrm{mEq} / \mathrm{L}$; Calcio: $7.5 \mathrm{mg} / \mathrm{dl}$; Magnesio: $1.5 \mathrm{mEq} / \mathrm{L}$ y acetato: 30 $\mathrm{mEq} / \mathrm{L}$.

La presión arterial, frecuencia cardiaca y frecuencia respiratoria prediálisis, fueron comparadas contra el punto más bajo o más alto que presentó la variación de la función vital durante la diálisis. Se estratificó la revolución de la presión arterial en tres categorías: incremento $(>20 \mathrm{mmHg}$ respecto al basal), caída $(<20 \mathrm{mmHg}$ respecto al basal) y no variación (si la presión se mantuvo en niveles de $\pm 19 \mathrm{mmHg}$ con respecto al basal durante toda la sesión).

El análisis de la asociación entre el tipo de baño y las variables dependientes discontínuas se hizo mediante chi cuadrado y prueba exacta de Fisher y ANOVA fue utilizado para análisis de varianza.

Los datos fueron analizados utilizando el software estadístico EPI INFO V5. 


\section{RESULTADOS}

La evolución de los parámetros gasométricos arteriales médicos $\left(\mathrm{pH}\right.$, bicarbonato, $\mathrm{pCO}_{2} \mathrm{y}$ $\mathrm{pO}_{2}$ ) pueden observarse comparativamente en las figuras $\mathrm{N}^{\circ} 1$ al $\mathrm{N}^{\circ} 4$. Los resultados se muestran como media aritmética y 1 desviación Standard. Los niveles séricos de bicarbonato fueron menores desde la primera hora hasta el final de la diálisis en las diálisis con acetato $(p<0.01)$, en el control postdiálisis no hubo diferencia entre los dos grupos.
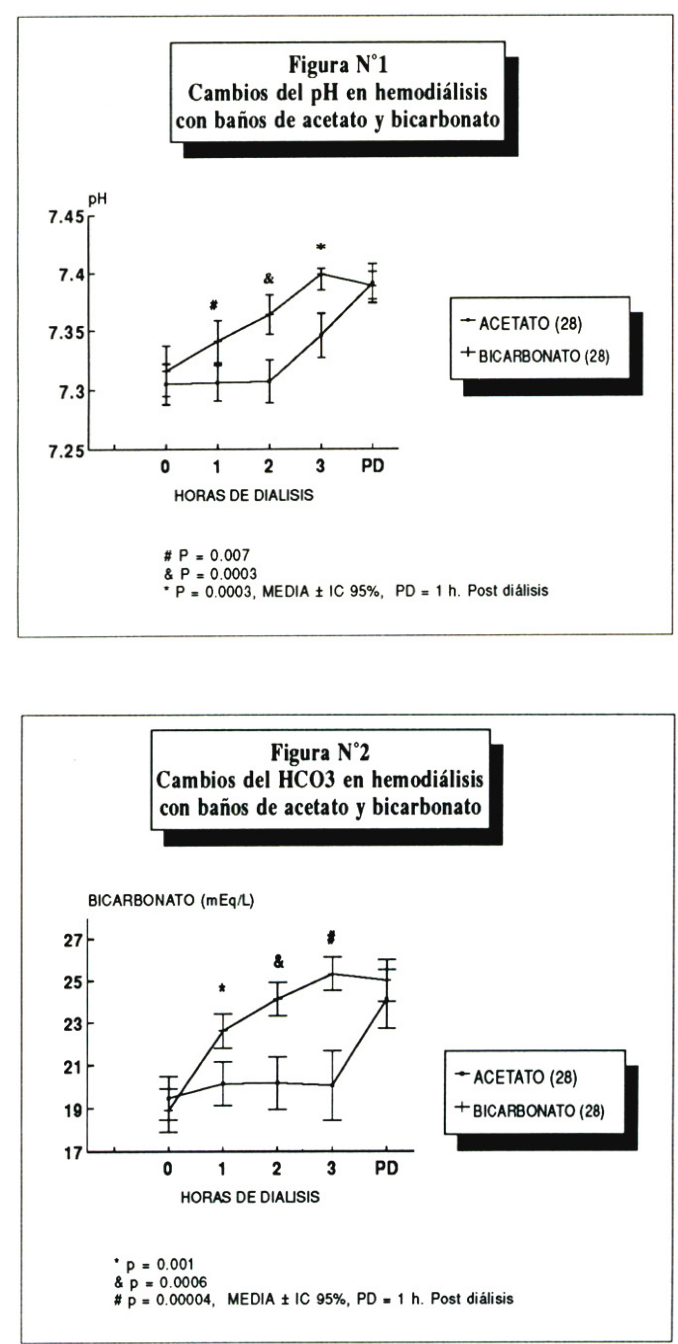

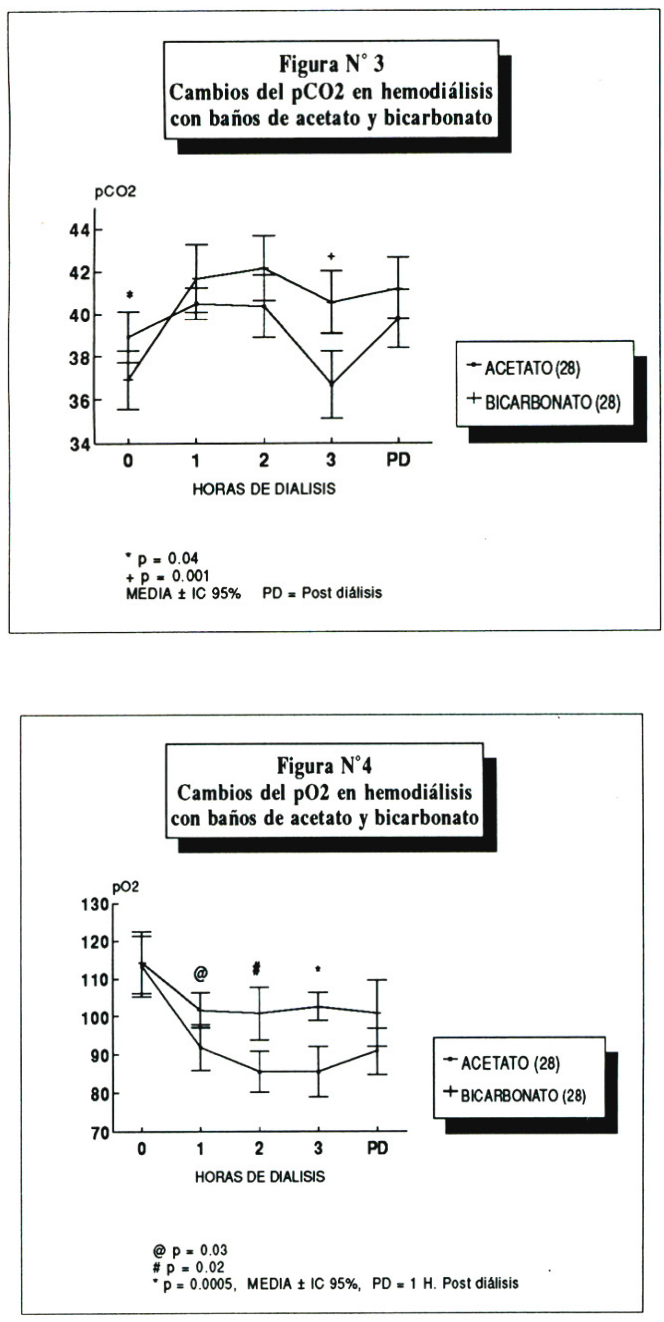

La $\mathrm{pCO}_{2}$ mostró una tendencia a ser mayor desde el inicio de los procedimientos en que se usó baño de bicarbonato. Pero sólo alcanzó significancia estadística a la tercera hora. En el control postdiálisis se observó una clara tendencia al incremento de la $\mathrm{pCO}_{2}$ de los pacientes dializados con acetato hasta valores similares a los del otro grupo.

También se encontraron mayores niveles de oxigenación arterial en los pacientes dializados con bicarbonato, la diferencia alcanzó significancia desde la primera hora hasta el final de la sesión $(\mathrm{p}<0.01)$, observándose que esta diferencia desapareció en el control postdiálisis.

El pH sérico del grupo dializado con bicarbonato fue significativamente mayor que el del grupo dializado con acetato durante toda la diálisis, no así en el control postdialítico.

Cefalea ocurrió en tres de las 28 sesiones de las diálisis con acetato, y en ninguno de las diálisis con bicarbonato ( $\mathrm{p}=\mathrm{ns}$ ). Náuseas y vómitos se presentaron en 10 de las 28 sesiones de las diálisis con acetato y en ninguna de las diálisis con bicarbonato $(p<0.05)$. Fue necesario infundir $\mathrm{ClNa} 0.9 \%$ en 7 de las 28 diálisis con baños de bicarbonato $(\mathrm{p}=\mathrm{ns})$ 
La frecuencia cardiaca y respiratoria durante las sesiones, tampoco mostraron diferencias en las diálisis con ambos baños durante el estudio no se observó reacción febril, ni sistémica que pudiera estar relacionada a los baños.

\section{DISCUSIÓN}

Los resultados de nuestro estudio confirman las ventajas de dializar con baños de bicarbonato en vez de acetato. La hipoxemia en hemodiálisis ha sido atribuida a la disminución del transporte de oxígeno a través de las membranas alveolares pulmonares, provocada por migración leucocitaria secundaria a su activación por membranas de diálisis nobiocompatibles $(12,13,14)$. También se le ha atribuido a hipoventilación alveolar causada por el efecto depresor del acetato sobre el centro respiratorio (15) y al mayor consumo de oxigeno indispensable para la oxidación de acetato en los hepatocitos y células musculares estriadas (16). A su vez, la hipoxemia causa inestabilidad hemodinámica al provocar vasodilatación sistémica $(6,16)$. Nuestros resultados, coincidentes con otros $(6$, 10), muestran que hubo menos hipoxemia cuando se usaron baños de bicarbonato. Considerando que este resultado no es atribuible a una membrana determinada, ya que nuestro estudio usa un mismo tipo de membrana, queda claro que la hipoxemia mostrada por el grupo de pacientes tratados con baño de acetato, depende fundamentalmente de la exposición de la sangre a este anión.

El pH y la concentración arterial de bicarbonato se incrementan significativamente desde el inicio en las diálisis con bicarbonato, mientras que en las de acetato ocurrieron después de la segunda y tercera hora. Los pacientes dializados con baños de bicarbonato mostraron niveles significativamente mayores y cercanos a los normales durante toda la sesión de diálisis.

El progresivo incremento del $\mathrm{pH}$ y bicarbonato de los pacientes dializados con baños de acetato, es consecuencia de la oxidación de este a bicarbonato en los hepatocitos y miocitos y explica la ausencia de diferencias en los controles tomados una hora post diálisis. Por su efecto vasodilatador sistémico, la academia es también un factor asociado a hipotensión en diálisis.

Los mayores niveles de $\mathrm{pCO}_{2}$ observados en el grupo dializado con bicarbonato se explican por la conversión de este buffer en $\mathrm{CO}_{2}$ y agua cuando reacciona con hidrogeniones libres. En el grupo dializado con acetato, el incremento de la $\mathrm{pCO}_{2}$ es más tardío y ocurre sólo después que una cantidad significativa de la masa de acetato transferida al paciente se transforma en bicarbonato.

La inestabilidad hemodinámica está asociada a ciertas características individuales de los pacientes como enfermedad de fondo, ganancia de peso interdialítica, tolerancia a cambios osmolales o de academia, dieta, compromiso cardiovascular, etc. El no haber encontrado disminución de los episodios de hipotensión arterial en el grupo estudiado cuando se usaron baños de bicarbonato, se podría explicar por otras condicionantes a la hipotensión arterial, diferentes a los cambios gasométricos producidos por el baño de bicarbonato. Resaltamos el hecho de que en otros estudios en los cuales se evaluaron poblaciones no seleccionadas, 
sí se ha observado una menor frecuencia de la hipotensión arterial sintomática con baños de bicarbonato $(17,18,19)$.

Sin embargo, es importante subrayar que molestias como náuseas y vómitos y cefalea desaparecieron por completo en las sesiones con bicarbonato.

Resulta incuestionable que la diálisis con baño con bicarbonato resulta fisiológicamente superior al de acetato, aún en diálisis convencionales con membranas de cuprophan. Sin embargo, esto no necesariamente se traduce en ventajas hemodinámicas para pacientes con gran sintomatología o ausencia de ella. Sí representa una ostensible mejoría en la frecuencia de náuseas y vómitos, manifestaciones relativamente frecuentes en las diálisis convencionales con acetato y que significa un alivio muy importante para el paciente.

\section{Agradecimiento:}

Este trabajo se realizó con el apoyo económico del Centro Privado de Hemodiálisis Servicios Médicos Córpac SA.

\section{Correspondencia:}

Dr. Javier Cieza Cevallos

Servicios Médicos Corpac. Los Cisnes 256. San Isidro. Lima.

\section{REFERENCIAS BIBLIOGRAFICAS}

1.Castillo W. Evaluación integral de un Programa de Hemodiálisis Crónica. (Tesis Doctoral). Lima, Perú: Universidad Peruana Cayetano Heredia, 1991.77 pp.

2.Situ O. Sobrevida de pacientes en Hemodiálisis Crónica Intermitente. (Tesis Doctoral). Lima, Perú: Universidad Peruana Cayetano Heredia, 1989. 60pp.

3.Held P, Pauly MV, Diamond LH. Survival analysis of patients undergoing dialysis. JAMA $1987 ; 257: 645-58$.

4.Held P, Brunner F, Odaka M, et al. Five-Year Survival for End-Stage Renal Disease Patients in the United States, Europe, and Japan, 1982 to 1987. Am J Kidney Dis 1990; 9:451-57.

5.Campistol JM, Solé M y Muñoz-Gómez J. Amiloidosis asociada a la diálisis. Nefrología 1990; 10 (Supl 3): 23-27.

6.Sargent J, Gotch F. Principles and biophisics of diálisis. En Replacement of Renal Function by Dialysis. A textbook of dialysis. Maher JF (ed). Kluwer Academic Publishers, 1986; Inc 67-68.

7.Heneghan WF. Acetate, Bicarbonate and Hypotension during hemodialysis. Am J Kidney Dis 1982; 2:302-04.

8.Van Stone JC. Hemodialysis. Year Book Medical Publishers, 1986; Inc 67-68.

9.Graefe U; Milutinovich J; Follete WC et al. Less Diálisis-Induced Morbidity and Vascular Instability with Bicarbonate in Dialysate. Ann Intern Med 1973; 88:332-36.

10.Hakim R; Pontzer MA; Tilton D; et al. Effects of acetate and bicarbonate dialysate in stable chronic dialysis patients. Kidney Int. 1985; 28:535-40. 
11.Kinet JP; Soyeur D; Balland N; et al. Hemodynamic study of hypotension during hemodialysis. Kidney Int 1982; 21:868-76.

12.Eiser AR. Pulmonary Gas Exchange during Hemodialysis and Peritoneal Dialysis: Interaction Between Respiration and Metabolism. Am J Kidney Dis 1985; 3: 131-42.

13.Francos G; Besarab A; Burke J; et al. Diálisis-Induced Hypoxemia: Membrana Dependent and Membrana Independent Causes. Am J Kidney Dis 1985; 3: 191-98.

14.Vaziri N; Wilson A; Mukai D; et al. Dialysis Hypoxemia : Role of Dialyzer Membrane and Dialysate Delivery System. Am J Med 1984; 77:828-33.

15.Dolan MJ; Whipp BJ; Davidson WP; et al. Hypopnea associated with acetate Hemodialysis: Carbon dioxide-Flow-dependent ventilation. N Engl J Med 1981; 305:72-75. 16.Pagel M; Ahmad S; Vizzo J; Scribner B. Acetate and bicarbonate fluctuations and acetate intolerance during dialysis. Kidney Int 1982; 21:513-18.

17.Valderrabano F. Tolerancia a la diálisis: factores implicados. Nefrología 1990 10(Supl 3): 44-53.

18.Vera Lora G. Santibáñez V. Batillana C.: Estudio Comparativo del equilibrio ácido-base y sintomatología en pacientes dializados con baños de acetato vs bicarbonato utilizando filtros de mediana eficiencia. Libro de Resúmenes, III Congreso Peruano de Nefrología pp: 28; 1984. Lima, Perú.

19.Ruder MA, Alpert MA, Van Stone J. Acetate and bicarbonato hemodiálisis in patients with and without autonomic dysfuction. Kidney Int 1990; 27: 768-71. 